\title{
ON THE ELEMENTARY THEORY OF PAIRS OF REAL CLOSED FIELDS. II
}

\author{
WALTER BAUR
}

§0. Introduction. Let $\mathscr{L}$ be the first order language of field theory with an additional one place predicate symbol. In [B2] it was shown that the elementary theory $T$ of the class $\mathscr{R}$ of all pairs of real closed fields, i.e., $\mathscr{L}$-structures $\langle K, L\rangle$, $K$ a real closed field, $L$ a real closed subfield of $K$, is undecidable.

The aim of this paper is to show that the elementary theory $T_{s}$ of a nontrivial subclass of $\mathscr{R}$ containing many naturally occurring pairs of real closed fields is decidable (Theorem 3, §5). This result was announced in [B2]. An explicit axiom system for $T_{s}$ will be given later. At this point let us just mention that any model of $T_{s}$ is elementarily equivalent to a pair of power series fields $\left\langle R_{0}\left(\left(T^{A}\right)\right), R_{1}\left(\left(T^{B}\right)\right)\right\rangle$ where $R_{0}$ is the field of real numbers, $R_{1}=R_{0}$ or the field of real algebraic numbers, and $B \subseteq A$ are ordered divisible abelian groups. Conversely, all these pairs of power series fields are models of $T_{s}$.

Theorem 3 together with the undecidability result in [B2] answers some of the questions asked in Macintyre [M]. The proof of Theorem 3 uses the model theoretic techniques for valued fields introduced by $\mathrm{Ax}$ and Kochen [A-K] and Ershov [E] (see also $[\mathrm{C}-\mathrm{K}]$ ). The two main ingredients are

(i) the completeness of the elementary theory of real closed fields with a distinguished dense proper real closed subfield (due to Robinson [R]),

(ii) the decidability of the elementary theory of pairs of ordered divisible abelian groups (proved in $\S \S 1-4$ ).

I would like to thank Angus Macintyre for fruitful discussions concerning the subject. The valuation theoretic method of classifying theories of pairs of real closed fields is taken from [M].

§1. Pairs of ordered groups. By a pair of ordered groups $\mathfrak{A}=\langle A, B\rangle$ we mean an ordered abelian group $A$ together with a distinguished subgroup $B$. Our first goal is the following:

THEOREM 1. The elementary theory $P$ of pairs of divisible ordered abelian groups is decidable.

Remarks. 1. The language of $P$ of course is the language of ordered groups with an additional predicate symbol for the distinguished subgroup.

2. The theory of pairs of ordered abelian groups (not necessarily divisible) is undecidable. A proof of this will be given at the end of $\S 4$.

Received July 17, 1980. 
From now on "group" means "divisible torsionfree abelian group". Thus a group is just a vector space over the rationals.

Definition. A pair of ordered groups $\mathfrak{A}=\langle A, B\rangle$ is called simple if

(D1) $A \neq 0$ and $B=0$, or

(D2) $A=B \neq 0$, or

(D3) $B$ is dense in $A$ and $B \neq A$.

It is well known (and easy to prove) that for each $i, 1 \leq i \leq 3$, the theory $P \cup(D i)$ is complete and hence decidable. The theory $\boldsymbol{P}$ will be reduced to the theories $P \cup(D i)$ and a suitable theory of ordered sets with distinguished subsets. Since the latter theory is also decidable Theorem 1 will follow.

We recall some notions from the theory of ordered groups. For more details see Fuchs [F]. A subgroup $C$ of an ordered group $A$ is called convex if for all $\gamma \in C$ and all $\alpha \in A,|\alpha|<|\gamma|$ implies $\alpha \in C$ where $|\alpha|=\max \{\alpha,-\alpha\} . C$ is principal, with generator $\gamma$ say, if $C$ is the smallest convex subgroup containing $\gamma$. If $C$ is convex then the ordering of $A$ induces an ordering of $A / C$ making $A / C$ into an ordered group. By a (convex) valuation of $A$ we mean a function $w$ from $A$ onto an ordered set $I$ with a maximal element $\infty$ such that (i) $w(\alpha)=\infty$ if and only if $\alpha=0$, (ii) $w(\alpha+\beta) \geq \min \{w(\alpha), w(\beta)\}$, (iii) $w$ is convex, i.e. for all $\alpha, \beta \in A$, $|\alpha| \leq|\beta|$ implies $w(\beta) \leq w(\alpha)$. For $u \in I$ put $A(u)=\{\alpha \in A \mid w(\alpha) \geq u\}$ and for $u \in I-\{\infty\}$ put $A_{>}(u)=\{\alpha \in A \mid w(\alpha)>u\} . A(u)$ and $A_{>}(u)$ are convex subgroups of $A$. Therefore the quotient groups $A(u) / A_{>}(u)$ are again ordered groups. If $w(\alpha)=u$ then $\alpha>0$ if and only if $\alpha+A_{>}(u)>0$ in $A(u) / A_{>}(u)$.

Now let $\mathfrak{A}=\langle A, B\rangle$ be a pair of ordered groups and $w: A \rightarrow I$ a valuation. For any subgroup $A^{\prime} \subseteq A$ and any convex subgroup $C \subseteq A$ put $\mathscr{A} \uparrow A^{\prime}=$ $\left\langle A^{\prime}, A^{\prime} \cap B\right\rangle$ and $\mathfrak{U} / C=\langle A / C,(B+C) / C\rangle$. Clearly both structures are pairs of ordered groups. Finally, for $u \in I-\{\infty\}$ put $\mathscr{Q}(u)=(\mathfrak{A} \mid A(u)) / A_{>}(u)$.

§2. The natural valuation. Let $\mathfrak{A}=\langle A, B\rangle$ be a pair of ordered groups and let $I$ be the set of principal convex subgroups of $A$. $I$ is ordered by inverse inclusion. Define $w: A \rightarrow I$ by $w(\alpha)=$ principal convex subgroup generated by $\alpha . w$ is a valuation of $A$, called the natural valuation (see [F]). All quotients $A(u) / A_{>}(u)$ are archimedean, i.e. isomorphic (as ordered groups) to subgroups of the reals. Therefore all quotients $\mathscr{2}(u)$ are simple.

For $\alpha \in A$ put $U(\alpha)=\{u \in I \mid \alpha \in A(u)+B\}$ and let $J$ be the smallest ordering extending $I$ such that $\sup U(\alpha)$ exists in $J$ for all $\alpha \in A$. Put $s(\alpha)=\sup U(\alpha)$. For $u \in J-I$ define $A(u)$ and $A_{>}(u)$ in the same way as for $u \in I$. Then $2(u)=0$ for $u \notin I$. Finally put

$$
L(u)=\left(\bigcap_{u^{\prime}<u}\left(A_{>}\left(u^{\prime}\right)+B\right)\right) /(A(u)+B) \quad(u \in J) .
$$

$(L(u)=0$ if $u=\min J$.) Note that $L(u) \neq 0$ for $u \in J-I . L(u)$ is just a group without ordering.

Now we are ready to associate with $\mathfrak{A}$ an ordering $\mathscr{T}(\mathfrak{U})$ with distinguished subsets as follows

$$
\mathscr{T}(\mathfrak{A})=\left\langle J ;\left\langle, I, S, P_{1}, P_{2}, P_{3}\right\rangle\right.
$$


where $J,<, I$ are defined as above and $S=\{u \in J \mid L(u) \neq 0\}$ and $P_{i}=\{u \in I \mid \mathscr{Q}(u)$ is simple of type $(D i)\}$.

We will show that the elementary theory of $\mathscr{T}(\mathfrak{A})$ determines the elementary theory of $\mathfrak{A}$.

§3. Elementary equivalence of pairs of ordered groups. We are going to axiomatize the situation of the last section. For this purpose we expand the language of pairs of ordered groups to a language appropriate for structures of the form $\mathfrak{A}^{+}=\langle\mathfrak{A}$, $w, \mathscr{T}(\mathfrak{A})>$ by adding predicate symbols for $w, J, I, S$ and the $P_{i}$. Let $P^{+}$be the theory (in the expanded language) with axioms expressing:

A1 $\mathfrak{A}$ is a pair of ordered groups;

A2 (i) $J$ is an ordered set (disjoint from $\mathfrak{A}$ ) with largest element $\infty$;

(ii) $I-\{\infty\}=P_{1} \cup \dot{\cup} P_{2} \cup P_{3}$ (disjoint union);

(iii) $J=I \cup S$;

(iv) $\forall s \in S\left[\exists u \in J(u<s) \& \forall u \in J\left(u<s \rightarrow \exists u^{\prime} \in P_{2} \cup P_{3}\left(u<u^{\prime}<s\right)\right)\right]$

A3 (i) $w$ is a convex valuation of $A$ onto $I$;

(ii) $\forall u \in I\left(u \in P_{i} \leftrightarrow \mathscr{Q}(u)\right.$ is simple of type $\left.(D i)\right)(i=1,2,3)$;

(iii) $\forall u \in J(u \in S \leftrightarrow L(u) \neq 0)$;

(iv) $\forall \alpha \in A(s(\alpha)=\sup U(\alpha)$ exists in $J)$.

REMARKs. 1. The quotient structures $\mathscr{Q}(u), L(u)$ and the set $U(\alpha)$ in A3 are defined with respect to the now arbitrary valuation $w$ just as they were defined before with respect to the natural valuation. It should be clear that A3 is indeed an elementary statement.

2. A2(iv) is a consequence of the remaining axioms.

By the construction described in the last section any pair $\mathfrak{A}$ of ordered groups has an expansion $\mathfrak{A}^{+}$to a model of $\boldsymbol{P}^{+}$. Therefore Theorem 1 follows from

THEOREM 2. $P^{+}$is decidable.

We need the following lemma which will be proved in the next section.

LEMMA 1. Let $\overline{\mathfrak{A}}=\langle\mathfrak{A}, w, \mathscr{T}\rangle, \overline{\mathfrak{A}}^{\prime}=\left\langle\mathfrak{A}^{\prime} w^{\prime}, \mathscr{T}^{\prime}\right\rangle$ be models of $P^{+}$. If $\mathscr{T}$ and $\mathscr{T}^{\prime}$ are elementarily equivalent then $\overline{\mathfrak{A}}$ and $\overline{\mathfrak{Q}}^{\prime}$ are elementarily equivalent.

Let $\mathscr{L}_{5}$ be the language of ordered sets with five distinguished subsets.

Corollary to Lemma 1. For any model $\overline{\mathfrak{A}}=\langle\mathfrak{A}, w, \mathscr{T}\rangle$ of $P^{+}$the set $P^{+} U$ $\mathrm{Th}_{\mathscr{L}_{5}}(\mathscr{T})$ is a complete axiom system for $\mathrm{Th}(\overline{\mathfrak{A}})$.

LEMMA 2. Let $\mathscr{T}$ be a countable $\mathscr{L}_{5}$-structure satisfying A2. Then there is a model $\overline{\mathfrak{A}}$ of $P^{+}$of the form $\overline{\mathfrak{A}}=\langle\mathfrak{A}, w, \mathscr{T}\rangle$.

Proof. Let $\mathscr{T}=\left\langle J ;\left\langle, I, S, P_{1}, P_{2}, P_{3}\right\rangle\right.$ and let $C=\Gamma_{u \in I^{\prime}} C_{u}$ be the lexicographic product over the ordered index set $I^{\prime}=I-\{\infty\}$ where $C_{u}=R$ for all $u \in I^{\prime}$. Let $A_{0}$ be the subgroup of $C$ of all elements of finite support and put

$$
B=\left\{c_{u_{1}}+\cdots+c_{u_{n}} \in A_{0} \mid n \in N, u_{i} \in P_{2} \cup P_{3}, c_{u_{i}} \in C_{u_{i}}, c_{u_{i}} \in Q \text { if } u_{i} \in P_{3}\right\} .
$$

For each $u \in S$ choose $\alpha_{u} \in C$ such that $\sup \left\{w\left(\alpha_{u}-\beta\right) \mid \beta \in B\right\}=u \notin\left\{w\left(\alpha_{u}-\beta\right) \mid\right.$ $\beta \in B\}$ where $w$ is the natural valuation of $C$. Finally put $A=A_{0}+\sum_{u \in S} \boldsymbol{Q} \alpha_{u}$ and $\mathfrak{A}=\langle A, B\rangle$. Clearly $\overline{\mathfrak{A}}=\langle\mathfrak{A}, w, \mathscr{T}\rangle$ is the required model.

Proof of Theorem 2. Since $P^{+}$is r.e. it suffices to show that $P^{+}$is co-r.e. Using compactness and Löwenheim-Skolem it follows from Lemma 2 and the Corollary to Lemma 1 that an arbitrary sentence $\varphi$ in the language of $P^{+}$is satisfiable in 
some model of $P^{+}$if and only if there exists an $\mathscr{L}_{5}$-sentence $\psi$ consistent with A2 such that $P^{+} \vdash \phi \rightarrow \varphi$. Therefore Theorem 2 follows from the fact that the $\mathscr{L}_{5^{-}}$ theory of ordered sets with five distinguished subsets is decidable (see [L-L]).

§4. Proof of Lemma 1. Let $\overline{\mathfrak{A}}, \overline{\mathfrak{A}}^{\prime}$ be $\boldsymbol{\aleph}_{0}$-saturated models of $P^{+}$satisfying the hypothesis of Lemma 1 . We show that $\overline{\mathfrak{A}}, \overline{\mathfrak{A}}{ }^{\prime}$ are partially isomorphic and hence elementarily equivalent.

Let $\mathscr{P}$ be the set of all pairs $\langle f, h\rangle$ such that:

(1) there exist finite-dimensional subspaces $A_{0}, A_{0}^{\prime}$ of $A, A^{\prime}$ such that $f$ is an isomorphism from $\mathfrak{A} \uparrow A_{0}=\left\langle A_{0}, B_{0}\right\rangle$ onto $\mathfrak{A}^{\prime}\left\lceil A_{0}^{\prime}=\left\langle A_{0}^{\prime}, B_{0}^{\prime}\right\rangle\right.$;

(2) $h$ is a partial elementary map from $\mathscr{T}$ into $\mathscr{T}^{\prime}$ (i.e. $\mathscr{T} \vDash \varphi(\mathbf{u}) \Leftrightarrow \mathscr{T}^{\prime} \vDash \varphi(h(\mathbf{u}))$ for all formulas $\varphi(\mathbf{x})$ and all $\mathbf{u}$ from $\operatorname{dom}(h))$ with finite domain containing $w\left(A_{0}\right) \cup$ $s\left(A_{0}\right)$

(3) for all $\alpha \in A_{0}$ :

(i) $h w(\alpha)=w^{\prime} f(\alpha)$,

(ii) $h s(\alpha)=s^{\prime} f(\alpha)$,

(iii) $s(\alpha) \in U(\alpha) \Leftrightarrow s^{\prime} f(\alpha) \in U^{\prime}(f(\alpha))$,

(iv) if $s(\alpha) \in U(\alpha)$ then $w(\alpha-\beta)=s(\alpha)$ for some $\beta \in B_{0}$;

(4) for all $u \in \operatorname{dom}(h)$ : the partial map $f_{u}$ from $\mathscr{Q}(u)$ into $\mathscr{Q}^{\prime}(h(u))$ induced by $f$ is elementary. (Note that $f_{u}$ is well defined by (3)(i).)

Since $\left\langle 0,\left\{\left\langle\infty, \infty^{\prime}\right\rangle\right\}\right\rangle$ is a member of $\mathscr{P}, \mathscr{P}$ is nonempty and it remains to prove the extension property. So let $\langle f, h\rangle \in \mathscr{P}$ and $n \in A \cup J-(\operatorname{dom}(f) \cup \operatorname{dom}(h))$. (The case $n^{\prime} \in A^{\prime} \cup J^{\prime}-(\operatorname{im}(f) \cup \operatorname{im}(h))$ is symmetric.) If $n \in J$ then, by (2) and $r_{0}$-saturation, there exists $n^{\prime} \in J^{\prime}$ such that $h_{1}=h \cup\left\{\left\langle n, n^{\prime}\right\rangle\right\}$ is elementary. Using A3(i), (ii) and completeness of the theories $P \cup(D i), 1 \leq i \leq 3$, it follows that $\left\langle f, h_{1}\right\rangle$ satisfies (4), hence $\left\langle f, h_{1}\right\rangle \in \mathscr{P}$. Now let $n=\alpha_{1} \in A$. Put $A_{1}=A_{0}+Q \alpha_{1}$, $B_{1}=A_{1} \cap B$.

Case 1. $B_{1} \neq B_{0}$.

Choose $\beta \in B_{1}-B_{0}$ such that $u=w(\beta)$ is maximal.

Case 1.1. $w\left(\beta-\alpha_{0}\right) \leq u$ for all $\alpha_{0} \in A_{0}$.

Using $\aleph_{0}$-saturation, first choose $u^{\prime} \in J^{\prime}$ such that $\bar{h}=h \cup\left\{\left\langle u, u^{\prime}\right\rangle\right\}$ is elementary. (If $u \in \operatorname{dom}(h)$ then $\bar{h}=h$ of course.) Then choose $\beta^{\prime} \in B^{\prime} \cap A^{\prime}\left(u^{\prime}\right)$ such that $f_{u} \cup\left\{\left\langle\beta+A_{>}(u), \beta^{\prime}+A_{>}^{\prime}\left(u^{\prime}\right)\right\rangle\right\}$ is elementary. (If $u \notin \operatorname{dom}(h)$ then $f_{u}$ denotes the $O$-map $\mathscr{Q}(u) \rightarrow \mathscr{Q}^{\prime}\left(u^{\prime}\right)$.) Define $\bar{f}: A_{1} \rightarrow A^{\prime}$ by $f \subseteq \bar{f}$ and $\bar{f}(\beta)=\beta^{\prime}$. It is easily checked that $\langle\bar{f}, \bar{h}\rangle \in \mathscr{P}$. Since we will prove the analogous statement in the next case the proof is left to the reader.

Case 1.2. $w\left(\beta-\alpha_{0}\right)>u$ for some $\alpha_{0} \in A_{0}$.

Choose $\alpha_{0} \in A_{0}$ such that $v=w\left(\beta-\alpha_{0}\right)$ is maximal. Note that $v<s\left(\alpha_{0}\right) \notin U\left(\alpha_{0}\right)$, because otherwise $w\left(\beta_{1}\right)>u$ for some $\beta_{1} \in B_{1}-B_{0}$, by (3)(iv) (take $\beta_{1}=\beta-\beta_{0}$ where $\beta_{0} \in B_{0}$ such that $\left.w\left(\alpha_{0}-\beta_{0}\right)=s\left(\alpha_{0}\right)\right)$. Using $\kappa_{0}$-saturation choose $v^{\prime} \in J^{\prime}$ such that $\bar{h}=h \cup\left\{\left\langle v, v^{\prime}\right\rangle\right\}$ is elementary. Again using $\boldsymbol{r}_{0}$-saturation choose $\beta^{\prime} \in B^{\prime} \cap A^{\prime}\left(v^{\prime}\right)$ such that $f_{v} \cup\left\{\left\langle\left(\beta-\alpha_{0}\right)+A_{>}(v), \beta^{\prime}+A_{>}^{\prime}\left(v^{\prime}\right)\right\rangle\right\}$ is elementary. Finally choose $\beta^{\prime \prime} \in B$ such that $w^{\prime}\left(\beta^{\prime \prime}-f\left(\alpha_{0}\right)\right)>v^{\prime}$ and define $\bar{f}: A_{1} \rightarrow A^{\prime}$ by $f \subseteq \bar{f}, \vec{f}(\beta)=\beta^{\prime}+\beta^{\prime \prime}$. Choice of $\beta^{\prime \prime}$ is possible since $s\left(\alpha_{0}\right) \notin U\left(\alpha_{0}\right)$. Now we show that $\langle\bar{f}, \bar{h}\rangle \in \mathscr{P}$.

(3)(i): Let $\alpha_{2}=q \beta-\alpha \in A_{1}, \alpha \in A_{0}, q \in Q$. If $q=0$ then (3)(i) holds by hypothesis. Therefore assume $q \neq 0$. Since $w\left(\alpha_{2}\right) \leq v$ and $w\left(\beta-\alpha_{0}\right)=v$ 
by the choice of $v$ and $\alpha_{0}$ we obtain $w\left(\alpha_{2}\right)=w\left(q\left(\beta-\alpha_{0}\right)+\left(q \alpha_{0}-\alpha\right)\right)=$ $\min \left\{v, w\left(q \alpha_{0}-\alpha\right)\right\}$. On the other hand

$$
\begin{aligned}
w^{\prime}\left(\bar{f}\left(\alpha_{2}\right)\right) & =w^{\prime}\left(q\left(\beta^{\prime \prime}-f\left(\alpha_{0}\right)\right)+q \beta^{\prime}+f\left(q \alpha_{0}-\alpha\right)\right) \\
& =\min \left\{v^{\prime}, w^{\prime}\left(f\left(q \alpha_{0}-\alpha\right)\right)\right\}
\end{aligned}
$$

by the choice of $\beta^{\prime}$ and because $w^{\prime}\left(\beta^{\prime \prime}-f\left(\alpha_{0}\right)\right)>v^{\prime}$. Therefore $\bar{h} w\left(\alpha_{2}\right)=w^{\prime} \bar{f}\left(\alpha_{2}\right)$ and hence (3)(i). Next note that if $\alpha \in A$ and $\beta \in B$ then $U(\alpha+\beta)=U(\alpha)$ and $s(\alpha+\beta)=s(\alpha)$. This implies the remaining parts of (3) because $A_{1}$ is generated over $A_{0}$ by some element from $B$. (1), (2) and (4) are immediate consequences of (3) and the construction.

Case 2. $B_{1}=B_{0}$.

Put $u=\max \left\{s(\alpha) \mid \alpha \in A_{1}-A_{0}\right\}$.

Case 2.1. There exists $\alpha \in A_{1}-A_{0}$ such that $s(\alpha)=u \in U(\alpha)$. By extending $\langle f, h\rangle$ according to Case 1 we may assume that

(a) $w\left(\alpha-\beta_{0}\right)=u$ for some $\beta_{0} \in B_{0}$,

(b) $\forall \alpha_{0} \in A_{0} \cap\left(B+A_{>}(u)\right) \exists \beta_{0} \in B_{0} w\left(\alpha_{0}-\beta_{0}\right)>u$.

Replacing $\alpha$ by $\alpha-\beta_{0}$ as in (a) we may assume $w(\alpha)=u$. Choose $u^{\prime} \in J^{\prime}$ and $\alpha^{\prime} \in A^{\prime}\left(u^{\prime}\right)$ such that the two maps $\bar{h}=h \cup\left\{\left\langle u, u^{\prime}\right\rangle\right\}$ and $f_{u} \cup\left\{\left\langle\alpha+A_{\rangle}(u)\right.\right.$, $\left.\left.\alpha^{\prime}+A_{>}^{\prime}\left(u^{\prime}\right)\right\rangle\right\}$ are elementary. Define $\bar{f}$ by $f \subseteq \bar{f}$ and $\bar{f}(\alpha)=\alpha^{\prime}$.

Case 2.2. Not Case 2.1.

Choose $\alpha \in A_{1}-A_{0}$ such that $s(\alpha)=u$. Choose $\beta \in B$ such that

(a) $\forall \alpha_{0} \in A_{0} w\left(\alpha-\alpha_{0}\right)<w(\alpha-\beta)=v$,

(b) $\forall u_{0} \in \operatorname{dom}(h)\left(v \leq u_{0} \rightarrow u \leq u_{0}\right)$.

Again by extending $\langle f, h\rangle$ according to Case 1 we may assume $\beta \in A_{0}$ (and (b) still holds). Replacing $\alpha$ by $\alpha-\beta$ we may assume $w(\alpha)=v$. Using $\kappa_{0}$-saturation choose $u^{\prime}, v^{\prime} \in J^{\prime}$ and $\beta^{\prime} \in B^{\prime} \cap A^{\prime}\left(v^{\prime}\right)$ such that $\bar{h}=h \cup\left\{\left\langle v, v^{\prime}\right\rangle,\left\langle u, u^{\prime}\right\rangle\right\}$ and $f_{v} \cup\left\{\left\langle\alpha+A_{>}(v), \beta^{\prime}+A_{>}^{\prime}\left(v^{\prime}\right)\right\rangle\right\}$ are elementary. Finally choose $\gamma^{\prime} \in A_{>}^{\prime}\left(v^{\prime}\right)$ such that $\gamma^{\prime} \in \bigcap_{i<u^{\prime}}\left(A_{>}^{\prime}(t)+B^{\prime}\right)$ and $\gamma^{\prime} \notin A_{0}^{\prime}+A^{\prime}\left(u^{\prime}\right)+B$. This is possible because $u^{\prime} \in S^{\prime}$. Now define $\bar{f}$ by $f \subseteq \bar{f}, \bar{f}(\alpha)=\beta^{\prime}+\gamma^{\prime}$.

The verification of (1)-(4) for $\langle\bar{f}, \bar{h}\rangle$ in Cases 2.1 and 2.2 is left to the reader.

We close this section by proving Remark 2 after Theorem 1. Put $C=\bigoplus_{i \in \omega} C_{i}$ where each $C_{i}$ is an ordered cyclic group with generator $c_{i}$. As a subgroup of the lexicographic product $P=\Gamma_{i \in \omega} C_{i}=\Pi_{i \in \omega} C_{i}, C$ is an ordered group. For any subset $X \subseteq \omega$ define $a_{X} \in P$ by $a_{X}(i)=c_{i}$ if $i \in X$ and $a_{X}(i)=0$ otherwise. Let $\mathscr{S}$ be an infinite set of pairwise disjoint infinite subsets of $\omega$ and let $I_{1}, \ldots, I_{k} \subseteq \mathscr{S}$ be pairwise disjoint. Let $p$ be a prime number and let $D$ be the subgroup of $P$ generated by the elements $p^{j} a_{X}$, where $X \in I_{j}, 1 \leq j \leq k$. Finally let $B$ be an arbitrary subgroup of $D$ and put $A=C+D$ and $\mathscr{U}=\langle A, B\rangle$. It is easy to see that

$$
\left(\bigcap_{i \in \omega}\left(A(i)+p^{k} A\right)\right) / p^{k} A \cong \bigoplus_{1 \leq j \leq k}\left(Z / p^{j} Z\right)^{\left(\kappa_{j}\right)}
$$

where $\kappa_{j}=$ card $I_{j}$. Furthermore the quotient group on the left-hand side is definable in $\mathfrak{A}$ by means of

$$
a \in \bigcap_{i \in \omega}\left(A(i)+p^{k} A\right) \Leftrightarrow \forall a^{\prime} \in A-\{0\} \exists c \in p^{k} A\left(|a-c|<\left|a^{\prime}\right|\right) .
$$


Therefore, for any given pair $\langle G, H\rangle$ of countable abelian groups such that $p^{k} G=0$ we can find $I_{1}, \ldots, I_{k} \subseteq \mathscr{S}$ and $B$ as above such that $\langle G, H\rangle$ is definable in $\mathfrak{A}$. By [B1] this implies undecidability of the theory of pairs of ordered abelian groups.

\section{\$5. Separated pairs of real closed flelds.}

Definition. A pair of valued real closed fields is a structure $\mathscr{K}=\langle K, L, A, B, v\rangle$ such that $\langle K, L\rangle \in \mathscr{R}=$ class of all pairs of real closed fields, $v$ is a valuation of $K$ (now of course in the sense of field theory) with value group $A$ and $B=v\left(L^{\times}\right)$. The residue class fields of $K, L$ are denoted by $K_{v}, L_{v}$, or simply by $\underline{K}, L$ if there is no danger of confusion. $\underline{\mathscr{K}}$ denotes the pair of fields $\langle\underline{K}, \underline{L}\rangle$. If $v$ is given by its valuation ring $V$ we also write $K_{V}$ for $K_{v}$. As in the case of ordered groups $v$ is called convex if for all $a, a^{\prime} \in K,|a| \leq\left|a^{\prime}\right|$ implies $v\left(a^{\prime}\right) \leq v(a)$, i.e. if the valuation ring associated to $v$ is a convex subset of $K . \mathscr{R}^{+}$is the class of all pairs $\mathscr{K}$ of valued real closed fields such that $v$ is convex.

Remarks. 1. The groups $A, B$ occurring in $\mathscr{K}$ are divisible: If $a \in K, 0<a$, then $n v\left(a^{1 / n}\right)=v(a)$. Therefore $\langle A, B\rangle$ is a pair of ordered divisible groups.

2. If $v$ is convex then $\mathscr{K} \in \mathscr{R}$.

3. Let $\langle A, B\rangle$ be an arbitrary pair of ordered divisible groups and $\left\langle R_{0}, R_{1}\right\rangle \in \mathscr{R}$. Then $\mathscr{K}=\left\langle R_{0}\left(\left(T^{A}\right)\right), R_{1}\left(\left(T^{B}\right)\right), A, B, v\right\rangle \in \mathscr{R}^{+}$where $R_{0}\left(\left(T^{A}\right)\right)$ is the field of formal power series with coefficients in $R_{0}$ and exponents in $A$, and $v$ is the natural valuation (see e.g. [P]). Also $\mathscr{K} \cong\left\langle R_{0}, R_{1}\right\rangle$.

Definition. Let $\varphi(x)$ be the $\mathscr{L}$-formula

$$
\forall y(|x| \leq y \leq 2|x| \rightarrow \exists z \in L(y<z<y+1)),
$$

and for $\langle K, L\rangle \in \mathscr{R}$ let $V_{0}$ be the set of all $a \in K$ satisfying $\varphi(x)$.

LEMMA 3. $V_{0}$ is the largest convex valuation ring of $K$ such that $L_{V_{0}}$ is dense in $K_{V_{0}}$.

Proof. First we show

(1) if $a_{1} \in V_{0}$ and $0 \leq a_{2} \leq 2 a_{1}$ then $a_{2} \in V_{0}$. Let $a_{2} \leq y \leq 2 a_{2}$. Since $a_{1} \in V_{0}$ there exists $z_{1} \in L$ such that $a_{1}<z_{1}<a_{1}+1$ and since $a_{1} \leq a_{1}+y / 4 \leq 2 a_{1}$ there exists $z_{2} \in L$ such that $a_{1}+y / 4<z_{2}<a_{1}+y / 4+1$. Combining the inequations involving $z_{1}, z_{2}$ we obtain $y<4\left(z_{2}-z_{1}+1\right)<y+8$ and hence $y<$ $4\left(z_{2}-z_{1}+1\right)+q<y+1$ for some $q \in Q$. Since $z=4\left(z_{2}-z_{1}+1\right)+q \in L$ it follows that $a_{2}$ satisfies $\varphi(x)$, i.e. $a_{2} \in V_{0}$.

Since $a \in V_{0}$ if and only if $|a| \in V_{0}$, (1) implies that $V_{0}$ is a convex subgroup of the additive group of $K$. Furthermore $1 \in V_{0}$.

Let $a, a^{\prime} \in V_{0}$. We show $a a^{\prime} \in V_{0}$. Assuming $0<a^{\prime} \leq a$ it suffices to show $a^{2} \in V_{0}$, by (1). If $a \leq 1$ then $a^{2} \in V_{0}$, again by (1). If $1<a$ let $a^{2} \leq y \leq 2 a^{2}$. Then $a \leq \sqrt{y}$ $\leq 2 a$ so there exists $z_{1} \in L$ such that $\sqrt{y}<z_{1}<\sqrt{y}+1$ hence $0<z_{1}-\sqrt{y}<1$. Since $0<z_{1}\left(z_{1}-\sqrt{y}\right)<z_{1} \leq 2 a+1 \in V_{0}$, (1) yields $z_{1}\left(z_{1}-\sqrt{y}\right) \in V_{0}$. Hence there exists $z_{2} \in L$ such that $z_{1}\left(z_{1}-\sqrt{y}\right)<z_{2}<z_{1}\left(z_{1}-\sqrt{y}\right)+1$. So

$$
\sqrt{y}<z_{1}+\frac{1-z_{2}}{z_{1}}<\sqrt{y}+\frac{1}{z_{1}}
$$

and

$$
y<\left(z_{1}+\frac{1-z_{2}}{z_{1}}\right)^{2}<y+\frac{2 \sqrt{y}}{z_{1}}+\frac{1}{z_{1}^{2}}<y+3
$$


because $1<\sqrt{y}<z_{1}$. Adding a suitable $q \in Q$ to the expression between the $<-$ signs we find $z \in L$ such that $y<z<y+1$. Hence $a^{2} \in V_{0}$.

It follows from what we have shown so far that $V_{0}$ is a convex valuation ring of $K$. In order to prove that $L_{V_{0}}$ is dense in $K_{V_{0}}$ let $a, a^{\prime} \in V_{0}$ such that $0<a<a^{\prime}$ and $a \neq a^{\prime}$ modulo the maximal ideal of $V_{0}$. Then $\left(a^{\prime}-a\right)^{-1} \in V_{0}$ so there exists $z_{1} \in L$ such that $\left(a^{\prime}-a\right)^{-1}<z_{1}<\left(a^{\prime}-a\right)^{-1}+1$. Now $z_{1} \in V_{0}$ so $a z_{1} \in V_{0}$ hence there exists $z_{2} \in L$ such that $a z_{1}<z_{2}<a z_{1}+1$, i.e. $a<z_{2} z_{1}^{-1}<a+z_{1}^{-1}<a^{\prime}$.

Finally let $V_{1}$ be an arbitrary convex valuation ring of $K$ such that $L_{V_{1}}$ is dense in $K_{V_{1}}$. Let $a \in V_{1}$ and $|a| \leq y \leq 2|a|$. Then $y \in V_{1}$, by convexity, hence there exists $z \in L \cap V_{1}$ such that $y<\underline{z}<y+1$ where $y$ is the residue class of $y$ in $K_{V_{1}}$. Therefore $y<z<y+1$ and so $a \in V_{0}$.

Definition (CF. [B2]). Let $\mathscr{K}=\langle K, L, A, B, v\rangle \in \mathscr{R}^{+}$. A sequence $\left\langle a_{1}, \ldots, a_{n}\right\rangle$ of elements from $K$ is called $(\mathscr{K}-)$ separated if for all $b_{1}, \ldots, b_{n} \in L, v\left(\sum_{i} a_{i} b_{i}\right)=$ $\min _{i} v\left(a_{i} b_{i}\right)$. (As usual $v(0)=\infty>A$.) $\mathscr{K}$ is called separated if any finite-dimensional $L$-vectorspace $\subseteq K$ has a separated basis.

Now we are ready to introduce the theory $T_{s}$ mentioned in the introduction: $T_{s}$ is the $\mathscr{L}$-theory of all pairs $\langle K, L\rangle \in \mathscr{R}$ such that $\left\langle K, L, v_{0}\left(K^{\times}\right), v_{0}\left(L^{\times}\right), v_{0}\right\rangle$ is separated where $v_{0}$ is the valuation with valuation ring $V_{0}$.

Clearly $T_{s}$ is axiomatizable. For each $n$ there is an axiom expressing separatedness for $n$-dimensional $L$-subspaces of $K$. Our main result is

THEOREM 3. 1. Two models $\langle K, L\rangle,\left\langle K^{\prime}, L^{\prime}\right\rangle$ of $T_{s}$ are elementarily equivalent if and only if

(i) $L_{V_{0}}=K_{V_{0}} \leftrightarrow L_{V_{0}^{\prime}}^{\prime}=K_{V_{0}^{\prime}}^{\prime}$, and

(ii) the associated pairs of value groups $\left\langle v_{0}\left(K^{\times}\right), v_{0}\left(L^{\times}\right)\right\rangle$and $\left\langle v_{0}\left(K^{\prime} \times\right), v_{0}\left(L^{\prime \times}\right)\right\rangle$ are elementarily equivalent as pairs of ordered groups.

2. Any pair of power series fields $\left\langle R_{0}\left(\left(T^{A}\right)\right), R_{1}\left(\left(T^{B}\right)\right)\right\rangle$ where $\langle A, B\rangle$ is a pair of ordered divisible abelian groups and $R_{0}=$ field of real numbers, $R_{1}=R_{0}$ or $=$ field of real algebraic numbers is a model of $T_{s}$. Any model of $T_{s}$ is elementarily equivalent to such a pair of power series fields.

3. $T_{s}$ is decidable.

The proof follows the same pattern as the proof of Theorem 1. It is convenient to expand the language $\mathscr{L}$ to a language $\mathscr{L}^{+}$appropriate for structures $\mathscr{K}=$ $\langle K, L, A, B, v\rangle \in \mathscr{R}^{+}$by adjoining symbols for the valuation and the value groups.

Let $T^{+}$be the $\mathscr{L}^{+}$-theory with axioms expressing

(i) $\mathscr{K}=\langle K, L, A, B, v\rangle \in \mathscr{R}^{+}$,

(ii) $\mathscr{K}$ is separated,

(iii) $L_{v}$ is dense in $K_{v}$.

The crucial step in the proof of Theorem 3 is the following lemma whose proof is postponed to the next section.

LemMA 4. Let $\mathscr{K}=\langle K, L, A, B, v\rangle, \mathscr{K}^{\prime}=\left\langle K^{\prime}, L^{\prime}, A^{\prime}, B^{\prime}, v^{\prime}\right\rangle$ be models of $T^{+}$ such that

(i) $\underline{L}=\underline{K} \Leftrightarrow \underline{L}^{\prime}=\underline{K}^{\prime}$, and

(ii) the pairs of value groups $\langle A, B\rangle$ and $\left\langle A^{\prime}, B^{\prime}\right\rangle$ are elementarily equivalent.

Then $\mathscr{K}$ and $\mathscr{K}^{\prime}$ are elementarily equivalent.

An immediate consequence of Lemma 4 is

COROLlaRY 1. Any model of $T^{+}$is elementarily equivalent to a valued pair of the 
form $\left\langle R_{0}\left(\left(T^{A}\right)\right), R_{1}\left(\left(T^{B}\right)\right), A, B, v\right\rangle$ where $R_{0}, R_{1}, A, B$ are as in Theorem 3 and $v$ is the natural valuation of $R_{0}\left(\left(T^{A}\right)\right)$. Conversely any valued pair of power series fields of this special form is a model of $T^{+}$.

Proof. It follows from [B2, Lemma 3] that any valued pair of power series fields as in the corollary is a model of $\mathrm{T}^{+}$.

Combining Lemma 4, Corollary 1 and Theorem 1 we get

Corollary 2. $T^{+}$is decidable.

Proof of THeorem 3. If $\langle K, L\rangle \models T_{s}$ then $\left\langle K, L, v_{0}\left(K^{\times}\right), v_{0}\left(L^{\times}\right), v_{0}\right\rangle \vDash T^{+}$. Conversely, if $\langle K, L, A, B, v\rangle \vDash T^{+}$then $\left\langle K, L, v_{0}\left(K^{\times}\right), v_{0}\left(L^{\times}\right), v_{0}\right\rangle \vDash T^{+}$because $V_{0}$ contains the valuation ring associated to $v$, by Lemma 3 , and hence $v_{0}\left(K^{\times}\right)$is a quotient of $A$. So $\langle K, L\rangle \vDash T_{s}$. Therefore the models of $T_{s}$ are precisely the $\mathscr{L}$-reducts of models of $T^{+}$. Theorem 3 now follows from Lemma 4 and its corollaries.

§6. Proof of Lemma 4. The following simple properties of separated sequences will be used: Let $\mathscr{K}=\langle K, L, A, B, v\rangle$ and $\sigma=\left\langle a_{1}, \ldots, a_{n}\right\rangle$ be a sequence of elements from $K$.

(S1) If $\sigma$ is separated then any subsequence of $\sigma$ is separated.

(S2) If $\sigma$ is separated and $b_{1}, \ldots, b_{n} \in L$ then $\left\langle a_{1} b_{1}, \ldots, a_{n} b_{n}\right\rangle$ is separated.

(S3) If $\sigma$ is separated and $a \in K$ then $\left\langle a a_{1}, \ldots, a a_{n}\right\rangle$ is separated.

(S4) If $v\left(a_{i}\right)=0,1 \leq i \leq n$, then $\sigma$ is separated if and only if $\underline{a}_{1}, \ldots, a_{n}$ are linearly independent over $L$.

(S5) If $\sigma$ is separated and $\left\langle a_{n+1}, \ldots, a_{m}\right\rangle$ is another separated sequence such that for all $i, j, 1 \leq i \leq n$, and $n<j \leq m$ implies $a_{i} a_{j}=0$ or $v\left(a_{i}\right) \neq v\left(a_{j}\right) \bmod B$ then $\left\langle a_{1}, \ldots, a_{m}\right\rangle$ is separated.

(S1), (S2) and (S3) are immediate consequences of the definition.

Proof of (S4). "Only if": Obvious.

"If": Assume $\sigma$ not separated and let $b_{1}, \ldots, b_{n} \in L$ such that $v\left(\sum_{i} a_{i} b_{i}\right)>$ $\min _{i} v\left(a_{i} b_{i}\right)$. Multiplying by some $b \in L$ we may assume $\min _{i} v\left(b_{i}\right)=0$. Then $\sum_{i} \underline{a}_{i} \underline{b}_{i}=0$ hence $\underline{a}_{1}, \ldots, \underline{a}_{n}$ are linearly dependent over $L$.

Proof of (S5).

$$
v\left(\sum_{1 \leq i \leq m} a_{i} b_{i}\right)=\min \left(v\left(\sum_{1 \leq i \leq n} a_{i} b_{i}\right), \quad v\left(\sum_{n+1 \leq i \leq m} a_{i} b_{i}\right)\right)=\min _{1 \leq i \leq m} v\left(a_{i} b_{i}\right) .
$$

Definition. Let $\mathscr{K}_{i}=\left\langle K_{i}, L_{i}, A_{i}, B_{i}, v_{i}\right\rangle \in \mathscr{R}^{+}, i=0,1, \mathscr{K}_{0}$ a substructure of $\mathscr{K}_{1}$, i.e. $K_{0} \subseteq K_{1}, v_{0}=v_{1} \uparrow K_{0}, L_{0}=K_{0} \cap L_{1}, B_{0}=A_{0} \cap B_{1} . \mathscr{K}_{0}$ is called an admissible substructure of $\mathscr{K}_{1}$ if $\underline{K}_{0}$ and $\underline{L}_{1}$ are linearly disjoint over $\underline{L}_{0}$. (In particular $\underline{K}_{0} \cap \underline{L}_{1}=\underline{L}_{0}$.)

LeMmA 5. Let $\mathscr{K}_{0}, \mathscr{K}_{1} \models T^{+}, \mathscr{K}_{0}$ an admissible substructure of $\mathscr{K}_{1}$.

(i) Any $\mathscr{K}_{0}$-separated sequence $\left\langle a_{1}, \ldots, a_{n}\right\rangle$ from $K_{0}$ is $\mathscr{K}_{1}$-separated.

(ii) $K_{0}$ and $L_{1}$ are linearly disjoint over $L_{0}$.

(i) is proved by induction on $n$. The cases $n=1$ or $a_{n}=0$ are trivial. Assume wlog that for some $k, 1 \leq k \leq n, v\left(a_{n}\right)=v\left(a_{i}\right) \bmod B_{1}$ if and only if $i<k$. Since $B_{0}=A_{0} \cap B_{1}$ there exist $b_{1}, \ldots, b_{k-1} \in L_{0}$ such that $v\left(a_{n}\right)=v\left(a_{i} b_{i}\right), 1 \leq i<k$. By (S1), (S2) the sequence $\left\langle a_{1} b_{1}, \ldots, a_{k-1} b_{k-1}, a_{n}\right\rangle$ is $\mathscr{K}_{0}$-separated. Therefore, by (S3), $\sigma=\left\langle a_{1} b_{1} / a_{n}, \ldots, a_{k-1} b_{k-1} / a_{n}, 1\right\rangle$ is $\mathscr{K}_{0}$-separated. $\sigma$ satisfies the hypothesis of (S4). Using linear disjointness it follows from (S4) that $\sigma$ is $\mathscr{K}_{1}$-separated. 
Again using (S3), (S2) we conclude that $\left\langle a_{1}, \ldots, a_{k-1}, a_{n}\right\rangle$ is $\mathscr{K}_{1}$-separated. Applying the induction hypothesis to $\left\langle a_{k}, \ldots, a_{n-1}\right\rangle$ we obtain, by (S5), that $\left\langle a_{1} \ldots, a_{n}\right\rangle$ is $\mathscr{K}_{1}$-separated.

(ii) Let $a_{1}, \ldots, a_{n} \in K_{0}$ be linearly independent over $L_{0}$. Since $\mathscr{K}_{0} \models T^{+}$the $L_{0}$-space $\Sigma_{i} L_{0} a_{i}$ has a $\mathscr{K}_{0}$-separated basis $\left\langle a_{1}^{\prime}, \ldots, a_{n}^{\prime}\right\rangle$. By (i) this sequence is $\mathscr{K}_{1}$-separated and hence linearly independent over $L_{1}$. Therefore $a_{1}, \ldots, a_{n}$ are linearly independent over $L_{1}$.

LEMMA 6. Let $\langle K, A, v\rangle,\left\langle K^{\prime}, A^{\prime}, v^{\prime}\right\rangle$ be valued real closed fields, $v, v^{\prime}$ convex. Let $f: K \rightarrow K^{\prime}$ and $F: A \rightarrow A^{\prime}$ be isomorphisms such that for some subfield $L$ of of $K, v\left(L^{\times}\right)=A$ and $v^{\prime} f\left\lceil L=F v\left\lceil L\right.\right.$. Then $v^{\prime} f=F v$.

Proof. Put $v_{1}=F v, v_{2}=v^{\prime} f$. Both $v_{1}$ and $v_{2}$ are convex valuations of $K$ and $v_{1}\left\lceil L^{\times}=v_{2}\left\lceil L^{\times}\right.\right.$. Assume $v_{1}(a)<v_{2}(a)$ for some $a \in K$. Choose $b \in L$ such that $v_{1}(a)<v_{1}(b)=v_{2}(b)<v_{2}(a)$. Then $|b|<|a|<|b|$ by convexity, contradiction.

LEMMA 7. Let $\langle K, A, v\rangle,\left\langle K^{\prime}, A^{\prime}, v^{\prime}\right\rangle$ be $\aleph_{1}$-saturated valued real closed fields, $v, v^{\prime}$ convex. Let $K_{0} \subseteq K_{1} \subseteq K$ be countable real closed subfields and let $f_{0}: K_{0} \rightarrow K^{\prime}$, $g: \underline{K}_{1} \rightarrow K^{\prime}, F: v\left(K_{1}^{\times}\right) \rightarrow A^{\prime}$ be isomorphic embeddings such that $v^{\prime} f_{0}=F v \backslash K_{0}$ and $g \mid \underline{K}_{0}=f_{0}$ where $f_{0}$ is the induced map $\underline{K}_{0} \rightarrow \underline{K}^{\prime}$. Then there exists an isomorphic embedding $f_{1}: K_{1} \rightarrow K^{\prime}$ extending $f_{0}$ such that $v^{\prime} f_{1}=F v\left\lceil K_{1}\right.$ and $g=f_{1}$.

This is a very special case of the main step in the usual model theoretic proofs of the Ax-Kochen-Ershov theorem on henselian fields (see e.g. [A], or [C-K, p. 271ff]). Details are left to the reader.

ProOF OF Lemma 4. Let $\mathscr{K}, \mathscr{K}^{\prime}$ be $\aleph_{1}$-saturated models of $T^{+}$satisfying the hypothesis of Lemma 4 . We show that they are partially isomorphic. Let $I$ be the set of all pairs $\langle f, F\rangle$ such that:

(i) $\langle f, F\rangle$ is an isomorphism from a countable admissible substructure $\mathscr{K}_{0} \models T^{+}$ of $\mathscr{K}$ onto an admissible substructure $\mathscr{K}_{0}^{\prime}$ of $\mathscr{K}^{\prime}$;

(ii) $F$ is a partial elementary map from $\langle A, B\rangle$ to $\left\langle A^{\prime}, B^{\prime}\right\rangle$;

(iii) the induced partial map $f$ from $\underline{\mathscr{K}}$ to $\underline{\mathscr{K}}^{\prime}$ is elementary.

REMARK. It follows from Robinson's proof [R] that (i) implies (iii). We will not use this fact.

$I \neq \varnothing:$ Let $K_{0}\left(K_{0}^{\prime}\right.$ resp.) be the algebraic closure of $Q$ in $K$ (in $K^{\prime}$ resp.) and let $f: K_{0} \rightarrow K_{0}^{\prime}$ be the unique isomorphism. Clearly $\langle f, 0\rangle \in I$.

$I$ has the extension property: Let $\left\langle f_{0}, F_{0}\right\rangle \in I$ be an isomorphism from $\mathscr{K}_{0}$ onto $\mathscr{K}_{0}^{\prime}$ and assume $a_{1} \in K-K_{0}$. (The case $a_{1}^{\prime} \in K^{\prime}-K_{0}^{\prime}$ is symmetric.)

Choose a countable elementary substructure $\mathscr{K}_{1}=\left\langle K_{1}, L_{1}, A_{1}, B_{1}, v \nmid K_{1}\right\rangle$ of $\mathscr{K}$ such that $K_{0} \cup\left\{a_{1}\right\} \subseteq K_{1}$. Obviously $\mathscr{K}_{1} \vDash T^{+}$is admissible. First choose an extension $F: A_{1} \rightarrow A^{\prime}$ of $F_{0}$ such that $F$ is elementary as a partial map from $\langle A, B\rangle$ to $\left\langle A^{\prime}, B^{\prime}\right\rangle$. Next choose an extension $g: K_{1} \rightarrow K^{\prime}$ of $f_{0}$ such that $g$ is elementary as a partial map from $\underline{\mathscr{K}}$ to $\underline{\mathscr{K}}^{\prime}$. Now apply Lemma 7 to get an extension $f_{1}: L_{1} \rightarrow$ $L^{\prime}$ of $f_{0}\left\lceil L_{0}\right.$ such that $F v\left\lceil L_{1}=v^{\prime} f_{1}\right.$ and $f_{1}=g\left\lceil L_{1}\right.$. Put $L_{1}^{\prime}=\operatorname{im}\left(f_{1}\right)$.

By Lemma $5, K_{0}$ and $L_{1}\left(K_{0}^{\prime}\right.$ and $L_{1}^{\prime}$ resp.) are linearly disjoint over $L_{0}$ (over $L_{0}^{\prime}$ resp.). Therefore $f_{1}$ extends to a field isomorphism $f_{2}: K_{0} L_{1} \rightarrow K_{0}^{\prime} L_{1}^{\prime}$ such that $f_{2} \uparrow K_{0}=f_{0}$.

Claim. (a) $v^{\prime} f_{2}=F v \mid K_{0} L_{1}\left(\right.$ and $\left.v\left(\left(K_{0} L_{1}\right)^{\times}\right)=A_{0}+B_{1}\right)$,

(b) $f_{2}=g \nmid K_{0} L_{1}$,

(c) $f_{2}$ is order preserving. 
(a) Since $K_{0} L_{1}$ is the quotient field of the set of all elements of the form

$$
a=\sum_{1 \leq i \leq n} a_{i} b_{i}, \quad a_{i} \in K_{0}, \quad b_{i} \in L_{1},
$$

it suffices to prove $v^{\prime} f_{2}(a)=F v(a)$ for elements $a$ of this form. Furthermore, since $\mathscr{K}_{0}$ is separated, we may assume that the sequence $\left\langle a_{1}, \ldots, a_{n}\right\rangle$ is $\mathscr{K}_{0}$-separated and hence $\mathscr{K}$-separated, by admissibility. Since $f_{0}$ is an isomorphism the sequence $\left\langle f_{0}\left(a_{1}\right), \ldots, f_{0}\left(a_{n}\right)\right\rangle$ is $\mathscr{K}_{0}^{\prime}$-separated, hence $\mathscr{K}^{\prime}$-separated. Therefore

$$
v(a)=\min v\left(a_{i} b_{i}\right) \in A_{0}+B_{1}
$$

and

$$
\begin{aligned}
v^{\prime}\left(f_{2}(a)\right) & =v^{\prime}\left(\sum f_{0}\left(a_{i}\right) f_{1}\left(b_{i}\right)\right)=\min \left(v^{\prime} f_{0}\left(a_{i}\right)+v^{\prime} f_{1}\left(b_{i}\right)\right) \\
& =\min \left(F v\left(a_{i}\right)+F v\left(b_{i}\right)\right)=F v(a) .
\end{aligned}
$$

(b) Since $\underline{f}_{2}$ coincides with $g$ on $\underline{K}_{0}$ and $\underline{L}_{1}$ it suffices to show that $K_{0} L_{1} \subseteq \underline{K}_{0} \underline{L}_{1}$. Let $c \in K_{0} L_{1}, v(c)=0$. Write $c=a / a^{\prime}$ where $a, a^{\prime}$ are of the form (2). By (a) there exist $a_{0} \in K_{0}, b_{0} \in L_{1}$ such that $v\left(a_{0} b_{0}\right)=v(a)=v\left(a^{\prime}\right)$. Dividing both $a$, $a^{\prime}$ by $a_{0} b_{0}$ we may assume $v(a)=v\left(a^{\prime}\right)=0$ and $a, a^{\prime}$ are still of the form (2). Now it suffices to show that $a \in \underline{K}_{0} \underline{L}_{1}$ (and similarly $\underline{a}^{\prime} \in \underline{K}_{0} \underline{L}_{1}$ ). As above write $a=\sum_{1 \leq i \leq n} a_{i} b_{i}$ where $\left\langle a_{1}, \ldots, a_{n}\right\rangle$ is $\mathscr{K}$-separated, $a_{i} \in K_{0}, b_{i} \in L_{1}$. Dropping the summands $a_{j} b_{j}$ with $v\left(a_{j} b_{j}\right)>0$ we may assume $v\left(a_{i} b_{i}\right)=0$ for all $i$. Then $v\left(a_{i}\right)=-v\left(b_{i}\right) \in A_{0} \bigcap$ $B_{1}=B_{0}$ so there exist $b_{i}^{\prime} \in L_{0}$ such that $v\left(a_{i} b_{i}^{\prime}\right)=0$. Writing $a=\Sigma\left(a_{i} b_{i}^{\prime}\right)\left(b_{i} b_{i}^{\prime}-1\right)$ we conclude $\underline{a}=\sum\left(a_{i} b_{i}^{\prime}\right)\left(b_{i} b_{i}^{\prime-1}\right) \in \underline{K}_{0} \underline{L}_{1}$.

(c) Let $0<a \in K_{0} L_{1}$. By (a) $v\left(K_{0} L_{1}\right)$ is divisible, hence there exists $c \in K_{0} L_{1}$ such that $v\left(a c^{2}\right)=0$. Then $0<a c^{2}$ and so $0<a c^{2}$. Since $g$ is order preserving we obtain $0<g\left(a c^{2}\right)=\underline{f}_{2}\left(a c^{2}\right)$, by (b), hence $0<f_{2}\left(a c^{2}\right)$ and finally $0<f_{2}(a)$. The claim is proved.

From (c) it follows that $f_{2}$ extends to an embedding $f_{3}$ of the relative algebraic closure $M$ of $K_{0} L_{1}$ into $K^{\prime} . M$ is just a real closure of $K_{0} L_{1}$. By Lemma $6 v^{\prime} f_{3}=$ $F v \uparrow M$, and $f_{3}=g \nmid \underline{M}$ because $\underline{M}$ is a real closure of $K_{0} L_{1}$ and therefore the extension of $g\left\lceil K_{0} L_{1}\right.$ to $\underline{M}$ is unique. By Lemma $7 f_{3}$ extends to an embedding $f: K_{1} \rightarrow K^{\prime}$ such that $f=g$ and $v^{\prime} f=F v$. Put $K_{1}^{\prime}=f\left(K_{1}\right)$. In order to show $\langle f, F\rangle \in I$ it remains to prove

(d) $K_{1}^{\prime} \cap L^{\prime}=L_{1}^{\prime}$,

(e) $\mathscr{K}_{1}^{\prime}=\mathscr{K}^{\prime} \backslash K_{1}^{\prime}$ is an admissible substructure of $\mathscr{K}^{\prime}$.

(d) Assume there exists $a \in K_{1}-L_{1}$ such that $f(a) \in L^{\prime}$. Since $\mathscr{K}_{1} \vDash T^{+}$the $L_{1}$-vectorspace $L_{1}+L_{1} a$ has a $\mathscr{K}_{1}$-separated basis $\left\langle a_{1}, a_{2}\right\rangle$. Since $f\left(a_{1}\right), f\left(a_{2}\right) \in L^{\prime}$ we have $v\left(a_{1}\right), v\left(a_{2}\right) \in B_{1}$ hence there exist $b_{1}, b_{2} \in L_{1}$ such that $v\left(a_{1} b_{1}\right)=v\left(a_{2} b_{2}\right)=0$. $\left\langle a_{1} b_{1}, a_{2} b_{2}\right\rangle$ is $\mathscr{K}_{1}$-separated and so wlog $a_{1} b_{1} \notin \underline{L}_{1}=\underline{K}_{1} \cap \underline{L}$ ((S2), (S4)). Therefore $f\left(a_{1} b_{1}\right)=g\left(a_{1} b_{1}\right) \notin L^{\prime}$ contradicting $f\left(a_{1} b_{1}\right) \in L^{\prime}$.

(e) follows from the fact that $\mathscr{K}_{1}$ is admissible and $g$ is partial elementary. 


\section{REFERENCES}

[A] J. Ax, A metamathematical approach to some problems in number theory, Proceedings of Symposia in Pure Mathematics, vol. 20, American Mathematical Society, Providence, RI, 1971, pp. 161-190.

[A-K] J. Ax and S. KochEN, Diophantine problems over local fields. I, II, III, American Journal of Mathematics, vol. 87 (1965), pp.605-630, 631-648; Annals of Mathematics, vol. 83 (1966), pp. $437-456$.

[B1] W. BAUR, Undecidability of the theory of abelian groups with a subgroup, Proceedings of the American Mathematical Society, vol. 55(1976), pp. 125-128.

[B2] - , Die Theorie der Paare reell abgeschlossener Körper, l'Enseignement Mathématique (to appear).

[C-K] C.C. Chang and H.J. KeISLER, Model theory, North-Holland, Amsterdam, 1973.

[E] YU. L. ERSHOV, On the elementary theory of maximal normed fields, Doklady Akademie Nauk SSSR, vol. 165 (1965), Soviet Mathematics. Doklady, pp. 1390-1393.

[F] L. FuChs, Partially ordered algebraic systems, Pergamon Press, Oxford, 1963.

[L-L] H. LÄUCHLI and J. LEONARD, On the elementary theory of linear order, Fundamenta Mathematicae, vol. 59 (1966), pp. 109-116.

[M] A. Macintyre, Classifying pairs of real closed fields, Ph. D. Dissertation, Stanford University, 1968.

[P] A. PRestel, Lectures on formally real fields, IMPA, Rio de Janeiro, 1975.

[R] A. Robinson, Solution of a problem of Tarski, Fundamenta Mathematicae, vol. 47 (1959), pp. 179-204.

\footnotetext{
SEMINAR FOR APPLIED MATHEMATICS

UNIVERSITY OF ZURICH

ZURICH, SWITZERLAND
} 